Article

\title{
Bivariate Kumaraswamy Models via Modified FGM Copulas: Properties and Applications
}

\author{
Indranil Ghosh \\ Department of Mathematics and Statistics, University of North Carolina, Wilmington, NC 28403, USA; \\ ghoshi@uncw.edu
}

Received: 13 September 2017; Accepted: 26 October 2017; Published: 1 November 2017

\begin{abstract}
A copula is a useful tool for constructing bivariate and/or multivariate distributions. In this article, we consider a new modified class of FGM (Farlie-Gumbel-Morgenstern) bivariate copula for constructing several different bivariate Kumaraswamy type copulas and discuss their structural properties, including dependence structures. It is established that construction of bivariate distributions by this method allows for greater flexibility in the values of Spearman's correlation coefficient, $\rho$ and Kendall's $\tau$.
\end{abstract}

Keywords: bivariate Kumaraswamy distribution; copula based construction; Kendall's tau; dependence structures

\section{Introduction}

Over the last decade or so, there has been a growing interest in constructing various bivariate distributions and study their dependence structure. For an excellent survey on this, an interested reader is suggested to see Balakrishnan and Lai (2009) and the references therein. Of late, copula based methods of construction have also gained a considerable amount of attention, mainly due to their analytical tractability in the sense of discussing dependence structure between two dependent random variables. A copula is a multivariate distribution function whose marginals are uniform on $[0,1]$ (see Sklar (1959), Nelsen (2006) for further details). It couples or links the marginal distributions to their joint distribution. In order to obtain a bivariate/multivariate distribution function, one needs to simply combine two (in the bivariate case) and/or several marginal distribution functions with any copula function. Consequently, for the purpose of statistical modeling, it is desirable to have a plethora of copulas at one's disposal. One of the most important parametric family of copulas is the Farlie-Gumbel-Morgenstern (FGM, henceforth) family defined as $C(u, v)=u v[1+\theta(1-u)(1-v)]$, $(u, v) \in(0,1)$, where $\theta \in[-1,1]$. This family of copulas have the following properties. Such family is derived from so called Farlie-Gumbel-Morgenstern distributions considered by Morgenstern (1956) and Gumbel (1960) and further developed by Farlie (1960).

- Symmetry: $C(u, v)=C(v, u), \forall(u, v) \in[0,1]^{2}$, and have the lower and upper tail dependence coefficients equal to zero.

- It is positive quadrant dependent (PQD) for $\theta \in(0,1]$ and negative quadrant dependent (NQD) for $\theta \in[-1,0)$.

However, the major drawback of FGM copula is that the range of values of Spearman's correlation coefficient $(\rho)$ and Kendal's $(\tau)$ is $[-1 / 3,1 / 3]$ and $[-2 / 9,2 / 9]$, respectively. To overcome this limited nature of dependence, several authors proposed extensions of this family (for example, Bairamov and Kotz (2000), Rodriguez-Lallena and Ubeda-Flores (2004)). It is to be noted here that a good number of literary works are available for the FGM family and the associated dependence parameter. Huang and Kotz (1999) studied a polynomial type parameter extensions of the FGM 
bivariate distribution and have shown that the positive correlation between the marginal distributions can be increased up to 0.39 , while the maximal negative correlation remains at $-1 / 3$. Lai and Xie (2000) used uniform representation of the FGM bivariate distributions having positive quadrant dependence (henceforth, PQD) with the association parameter between 0 and 1. Bairamov and Kotz (2000) showed that, for such a bivariate family, the related association parameter has a much wider range. In another article, Bairamov et al. (2001) developed a new generalization of the bivariate FGM distribution by introducing additional parameters. In their representation, with some specific choice of the functions $A(x)=1-x$, and $B(y)=1-y$ (see Equation (1) of Bairamov et al. (2001), they have shown that the admissible range for the association parameter is between $[-1,1]$, while the Pearson correlation coefficient $\rho$ between $X$ and $Y$ will never exceed $1 / 3$.

This fuels working in this direction in the sense of considering a modified FGM class and using it as a pivot for constructing bivariate Kumaraswamy models.

The Kumaraswamy distribution (Kumaraswamy 1980) is a two parameter absolutely continuous distribution useful for double bounded random processes with hydrological applications. The Kumaraswamy distribution (hereafter the KW distribution) on the interval $(0,1)$ has its probability density function (pdf) and its cumulative distribution function (cdf) with two shape parameters $a>0$ and $b>0$ defined by

$$
f(x)=a b x^{a-1}\left(1-x^{a}\right)^{b-1}, \quad(0<x<1), \quad \text { and } \quad F(x)=1-\left(1-x^{a}\right)^{b} .
$$

If a random variable $X$ has Equation (1) as its density, then we will write $X \sim K W(\delta, \beta)$ (for details, see Jones (2009)). The density function in Equation (1) has similar properties to those of the beta distribution. The KW pdf is unimodal, uniantimodal, increasing, decreasing or constant depending (similar to the beta distribution) on the values of the parameters. However, the construction of bivariate KW distributions has received limited attention. Barreto-Souza and Lemonte (2013) introduced a bivariate KW distribution related to a Marshall-Olkin survival copula and discussed some structural properties of their bivariate KW distributions. Arnold and Ghosh (2017) discussed some different strategies for constructing legitimate bivariate KW models via Arnold-Ng type copula approach. Recently, Ghosh and Ray (2016) discussed some copula based approach to construct several bivariate KW type models along with an application to a real life data set focusing on financial risk assessment. This article is a follow up paper to Ghosh and Ray (2016), in which we examine in detail the utility of a well-known bivariate FGM copula by a slight modification to allow greater flexibility in modeling various types of data sets. In this article, we start with a standard KW quantile function from two independent KW distributions (with two different sets of shape parameters) and construct the corresponding bivariate copula with different shape parameters. The rest of the article is organized as follows: in Section 2, we define the modified FGM copula and discuss some structural properties. In Section 3, we consider four special classes of modified bivariate KW FGM type copulas for constructing bivariate KW distributions. In Section 4, we establish some dependence structures for those developed bivariate KW FGM type copulas. In Section 5, an outline of simulation from the proposed copula model is provided. In Section 6, some applications of the four bivariate KW-FGM type copula models on two real-life data insurance data sets are considered for illustrative purposes. In Section 7, some concluding remarks are presented.

\section{Modified Bivariate FGM Copula}

We consider the following modified version of the bivariate FGM copula defined as

$$
C(u, v)=u v[1+\theta \Phi(u) \Psi(v)]=u v+\theta \widetilde{\Phi(u)} \widetilde{\Psi(v)},
$$

where $\widetilde{\Phi(u)}=u \Phi(u)$, and $\widetilde{\Psi(v)}=v \Psi(v)$, and $\theta \in[-1,1]$.

For a detailed study on this family of bivariate copula, see Rodriguez-Lallena and Ubeda-Flores (2004), where $\Phi(u)$ and $\Psi(v)$ are two absolutely continuous functions on $(0,1)$ with the following conditions. 
- $\Phi(0)=\Psi(0)=\Phi(1)=\Psi(1)=0$. This is known as a boundary condition.

- $\min \{\alpha \beta, \xi \eta\} \geq 1$, where $\alpha=\inf \left\{\frac{\partial \widetilde{\Phi u}}{\partial u}: u \in A_{1}\right\}<0$,

where $\frac{\partial \widetilde{\Phi(u)}}{\partial u}=\Phi(u)+u \frac{\partial \Phi(u)}{\partial u}$,

$\beta=\sup \left\{\frac{\partial \widetilde{\Phi(u)}}{\partial u}: u \in A_{1}\right\}>0$.

Again, $\xi=\inf \left\{\frac{\partial \widetilde{\Psi(v)}}{\partial v}: v \in A_{2}\right\}<0$, and

$\eta=\sup \left\{\frac{\partial \Psi(v)}{\partial v}: v \in A_{2}\right\}>0$, where

$$
\begin{aligned}
& A_{1}=\left\{u \in(0,1): \frac{\partial \widetilde{\Phi(u)}}{\partial u} \text { exists }\right\} \\
& A_{2}=\left\{v \in(0,1): \frac{\partial \widetilde{\Psi(v)}}{\partial v} \text { exists }\right\} .
\end{aligned}
$$

Theorem 1. The function in Equation (2) is a valid copula provided, the functions $\widetilde{\Phi(u)}, \widetilde{\Psi(v)}$ satisfy all the conditions stated above. In addition, provided all the conditions are satisfied, the bivariate copula in Equation (2) is absolutely continuous.

Proof. The proof immediately follows, since it matches with the form of bivariate copula (Equation (3), p. 316) in Rodriguez-Lallena and Ubeda-Flores (2004).

First, we make a note of the following:

- The associated bivariate copula density from Equation (2) will be

$$
c(u, v)=\frac{\partial C(u, v)}{\partial u \partial v}=1+\theta \Phi(u) \Psi(v)\left\{\left[1+u \frac{\partial \Phi(u)}{\partial u}\right]\left[1+v \frac{\partial \Phi(v)}{\partial v}\right]\right\} .
$$

- The conditional copula density of $U$ given $V=v$, from Equation (3), will be

$$
c(u \mid v)=\frac{\partial C(u, v)}{\partial v}=u\{1+\theta \Phi(u) \Psi(v)(1+v)\} .
$$

Similarly, one can find the conditional copula density of $V$ given $U=u$.

It is noteworthy to mention that copulas are instrumental for understanding the dependence between random variables. With them, we can separate the underlying dependence from the marginal distributions. It is well known that a copula that characterizes dependence is invariant under strictly monotone transformations. Subsequently, a better global measure of dependence would also be invariant under such transformations. Among other dependence measures, Kendall's and Spearman's are invariant under strictly monotone transformations of the random variables, and, as we will see in the next section, they can be expressed in terms of the associated copula.

- Kendall's $\tau$ : This measures the amount of concordance present in a bivariate distribution. Suppose that $(X, Y)$ and $(\tilde{X}, \tilde{Y})$ are two independent pairs of random variables from a joint distribution function. We say that these pairs are concordant if "large values of one tend to be associated" with "large values of the other", and "small values of one" tend to be associated with "small values of the other". The pairs are called discordant if large goes with small or vice versa. Algebraically we have concordant pairs if $(X-\tilde{X})(Y-\tilde{Y})>0$ and discordant pairs if we reverse the inequality. Let $X$ and $Y$ be continuous random variables with copula $C$. Then, Kendall's $\tau$ is given by 


$$
\tau_{s}(X, Y)=4 \iint_{[0,1]^{2}} C(u, v) d C(u, v)-1 .
$$

- Spearman's $\rho$ : For two random variables, $X$ and $Y$ are equal to the linear correlation coefficient between $F_{1}(X)$ and $F_{2}(Y)$, where $F_{1}$ and $F_{2}$ are the marginal distributions of $X$ and $Y$, respectively. Then, Spearman's $\rho_{s}$ is given by

$$
\rho_{s}(X, Y)=\rho\left(U=F_{1}(X), V=F_{2}(Y)\right)=12 \iint_{[0,1]^{2}} u v d C(u, v)-3,
$$

where $\rho$ is the linear correlation coefficient.

Alternatively, $\rho_{s}(X, Y)$ can be written as $\rho_{s}=12 \int_{0}^{1} \int_{0}^{1}[C(u, v)-u v] d u d v$. Also, as mentioned earlier, one can equivalently show that $\rho_{s}(U, V)=\rho\left(F_{1}(X), F_{2}(V)\right)$. For details on such copula based measures of dependence, see Nelsen (2006).

Proposition 1. Let $(X, Y)$ be a random pair with copula $C(u, v)$ given by Equation (2). Then, the expressions for Kendall's tau and Spearman's rho are

- $\rho_{s}(X, Y)=\theta A(u, v)$, where $A(u, v)=12\left[\int_{0}^{1} u \Phi(u) d u\right]\left[\int_{0}^{1} v \Psi(v) d v\right]$,

$$
\begin{aligned}
\tau_{s}(X, Y)= & \frac{2}{3} \rho_{\theta}+\int_{0}^{1} v^{2} \frac{\Psi^{\prime}(v)}{\Psi(v)} d v\left\{2+\int_{0}^{1} u^{2} \frac{\Phi^{\prime}(u)}{\Phi(u)} d u\right\} \\
& +\theta^{2}\left\{\left(\int_{0}^{1} u \Phi^{\prime}(u) \Phi(u) d u\right)\left(\int_{0}^{1} v \Psi^{\prime}(v) \Psi(v) d v\right)\right\}
\end{aligned}
$$

respectively.

Proof. The proofs are almost similar in approach for the two coefficients. First, consider for the Spearman's $\rho_{s}(X, Y)$. For our copula model in Equation (2), the corresponding $\rho_{s}(X, Y)$ will be

$$
\begin{aligned}
\rho_{s}(X, Y) & =12 \int_{0}^{1} \int_{0}^{1} C(u, v) d u d v-3 \\
& =12\left[\int_{0}^{1} v\left(\int_{0}^{1} u(1+\theta \Phi(u) \Psi(v)) d u\right) d v\right]-3 .
\end{aligned}
$$

Next, consider the integral in parenthesis, which, after some simplification, reduces to

$$
\int_{0}^{1} u(1+\theta \Phi(u) \Psi(v)) d u=\frac{1}{2}+\theta \Psi(v) \int_{0}^{1} u \Phi(u) d u
$$

Substituting Equation (8) in Equation (7), we get

$$
\begin{aligned}
\rho_{s}(X, Y) & =12\left[\int_{0}^{1} v\left(\frac{1}{2}+\theta \Psi(v) \int_{0}^{1} u \Phi(u) d u\right)\right] d v-3 \\
& =\theta A(u, v)
\end{aligned}
$$

after simple algebraic operation-hence the result.

Next, for the proof of $\tau_{s}(X, Y)$, note that from Equations (2) and (3), one may write (by taking their product) 


$$
\begin{aligned}
C(u, v) c(u, v)= & u v+\theta u v \Phi(u) \Psi(v)+(\theta \Phi(u) \Psi(v))\left\{u\left[1+u \frac{\partial \Phi(u)}{\partial u}\right]\right\}\left\{v\left[1+v \frac{\partial \Phi(v)}{\partial v}\right]\right\} \\
& +\left(\theta^{2} u v \Phi^{2}(u) \Psi^{2}(v)\right) \Phi(u) \Psi(v)\left\{u\left[1+u \frac{\partial \Phi(u)}{\partial u}\right]\right\}\left\{v\left[1+v \frac{\partial \Phi(v)}{\partial v}\right]\right\} .
\end{aligned}
$$

Our result in the expression for $\tau_{s}(X, Y)$ immediately follows by substituting Equation (9) in Equation (5), and after some simple algebra-hence the result. In the next section, we will consider some specific choices of $\Phi(u)$ and $\Psi(v)$ to construct bivariate Kumaraswamy type copulas.

\section{Bivariate KW-FGM Type Models}

In this section, we discuss in detail two different types of bivariate FGM type copula models to construct bivariate KW-type distribution.

\section{Bivariate KW-FGM (Type I) Model:}

Here, we consider the following functional form for both $\Phi(u)$ and $\Psi(v)$ :

- $\Phi(u)=u\left(1-u^{a_{1}}\right)^{b_{1}}$, for $\left(a_{1}, b_{1}\right)>0$,

- $\quad \Psi(v)=v\left(1-v^{a_{2}}\right)^{b_{2}}$, for $\left(a_{2}, b_{2}\right)>0$.

Note that this particular functional form does satisfy all the conditions stated earlier for $\Phi(u)$ and $\Psi(v)$. In that case, the corresponding bivariate copula (obtained from Equation (2)) will be given by

$$
C(u, v)=u v\left[1+\theta\left(u\left(1-u^{a_{1}}\right)^{b_{1}}\right)\left(v\left(1-v^{a_{2}}\right)^{b_{2}}\right)\right] .
$$

Next, suppose $X_{1} \sim K W\left(\lambda_{1}, \alpha_{1}\right) X_{2} \sim K W\left(\lambda_{2}, \alpha_{2}\right)$ and they are independent. Then, using Equation (10), a bivariate dependent FGM-Kumaraswamy (Type I) distribution will be of the following form (replacing $u$ and $v$ by the quantiles of $X_{1}$ and $X_{2}$, respectively):

$$
\begin{aligned}
& F\left(x_{1}, x_{2}\right) \\
& =\left(1-\left(1-x_{1}^{\lambda_{1}}\right)^{\alpha_{1}}\right)\left(1-\left(1-x_{1}^{\lambda_{2}}\right)^{\alpha_{2}}\right) \\
& \times\left\{1+\theta\left(1-\left(1-x_{1}^{\lambda_{1}}\right)^{\alpha_{1}}\right)\left(1-\left(1-\left(1-x_{1}^{\lambda_{1}}\right)^{\alpha_{1}}\right)^{b_{1}}\right)\right. \\
& \left.\times\left(1-\left(1-x_{2}^{\lambda_{2}}\right)^{\alpha_{2}}\right)\left(1-\left(1-\left(1-x_{2}^{\lambda_{2}}\right)^{\alpha_{2}}\right)^{b_{2}}\right)\right\},
\end{aligned}
$$

for $\left(\lambda_{1}, \lambda_{2}, \alpha_{1}, \alpha_{2}\right)>0$ and $0<\left(x_{1}, x_{2}\right)<1$.

\section{Bivariate KW-FGM (Type II) Model:}

Here, we consider the following functional form for both $\Phi(u)$ and $\Psi(v)$ :

- $\Phi(u)=u^{\delta_{1}}(1-u)^{1-\delta_{1}}$, for $\delta_{1}>0$,

- $\Psi(v)=v^{\delta_{2}}(1-v)^{1-\delta_{2}}$, for $\delta_{2}>0$.

Note that this particular functional form does satisfy all the conditions stated earlier for $\Phi(u)$ and $\Psi(v)$. In that case, the corresponding bivariate copula (henceforth, BK-FGM(Type II) copula) will be given by

$$
C(u, v)=u v\left[1+\theta u^{\delta_{1}} v^{\delta_{2}}(1-u)^{1-\delta_{1}}(1-v)^{1-\delta_{2}}\right] .
$$


In this case, like the previous one, a bivariate dependent KW-FGM (Type II) distribution, arising from two independent $\mathrm{KW}$ variables, will be of the following form:

$$
\begin{aligned}
& F\left(x_{1}, x_{2}\right) \\
& =\left(1-\left(1-x_{1}^{\lambda_{1}}\right)^{\alpha_{1}}\right)^{\delta_{1}}\left(1-\left(1-x_{2}^{\lambda_{2}}\right)^{\alpha_{2}}\right)^{\delta_{2}} \\
& \times\left[1+\theta\left(1-\left(1-x_{1}^{\lambda_{1}}\right)^{\alpha_{1}}\right)^{\delta_{1}}\left(\left(1-x_{1}^{\lambda_{1}}\right)^{\alpha_{1}\left(1-\delta_{1}\right)}\right)\right. \\
& \left.\times\left(1-\left(1-x_{2}^{\lambda_{2}}\right)^{\alpha_{2}}\right)^{\delta_{2}}\left(\left(1-x_{2}^{\lambda_{2}}\right)^{\alpha_{2}\left(1-\delta_{2}\right)}\right)\right] .
\end{aligned}
$$

\section{Bivariate KW-FGM (Type III) Model:}

Here, we consider the following functional form for both $\Phi(u)$ and $\Psi(v)$ :

- $\Phi(u)=u[\log (1+(1-u))]$

- $\Psi(v)=v[\log (1+(1-v))]$.

Note that this particular functional form does satisfy all the conditions stated earlier for $\Phi(u)$ and $\Psi(v)$. In that case, the corresponding BK-FGM (Type III) copula will be given by

$$
C(u, v)=u v[1+\theta u v\{\log (1+(1-u)) \log (1+(1-v))\}] .
$$

In this case, one can also obtain a closed form expression for the associated distribution function.

\section{Bivariate KW-FGM (Type-IV) Copula:}

For the standard KW distribution with parameters $(a, b)$, we have the pdf, cdf and the inverse cdf are given, respectively, by

$$
f_{i}\left(x_{i}\right)=a b x_{i}^{a-1}\left(1-x_{i}^{a}\right)^{b-1}, F_{i}\left(x_{i}\right)=1-\left(1-x_{i}^{a}\right)^{b} \text { and } F_{i}^{-1}\left(u_{i}\right)=1-\left(1-u_{i}^{1 / b}\right)^{1 / a}, a>0, b>0 .
$$

Hence, the associated copula for suitable parameters $a$ and $b$, and having two given marginal distributions that are the standard KW distributions, has the following form:

$$
\begin{aligned}
C\left(u_{1}, u_{2}\right)= & u_{1}\left(1-\left(1-u_{2}\right)^{1 / b}\right)^{1 / a}+u_{2}\left(1-\left(1-u_{1}\right)^{1 / b}\right)^{1 / a} \\
& -\left(1-\left(1-u_{1}\right)^{1 / b}\right)^{1 / a}\left(1-\left(1-u_{2}\right)^{1 / b}\right)^{1 / a} .
\end{aligned}
$$

For details on this, see Ghosh and Ray (2016).

\section{Some Properties of the Bivariate KW-FGM Type Copulas}

Next, we have the following:

1. For the BK-FGM (Type I) bivariate copula

- Closed form expression for Kendall's $\tau$ is not available.

- Spearman's correlation coefficient will be

$$
\rho_{\theta}=\theta\left(a_{1} a_{2}\right)^{-1}
$$

provided $\max \left(a_{1}, a_{2}\right)<3$.

2. For the BK-FGM (Type II) bivariate copula

- Kendall's $\tau$ will be

$$
\begin{aligned}
\tau_{s}(X, Y)= & B\left(\delta_{1}+2,2-\delta_{1}\right) B\left(\delta_{1}+3,2-\delta_{1}\right)+\left(\delta_{1}-1\right)\left[B\left(\delta_{1}+2,2-\delta_{1}\right)-B\left(\delta_{1}+1,1-\delta_{1}\right)\right] \\
& +\delta_{1} B\left(\delta_{1}+2,1-\delta_{1}\right)\left(\delta_{1}-\frac{1}{2}\right)-\frac{B\left(\delta_{1}+1,1-\delta_{1}\right)}{2}
\end{aligned}
$$

provided $\delta_{1}<1$. 
- Corresponding Spearman's correlation coefficient will be

$$
\rho_{s}(X, Y)=\theta\left(B\left(\delta_{1}+2,2-\delta_{1}\right)\right)^{2}
$$

provided $\delta_{1}<2$.

3. For the BK-FGM (Type III) copula, no closed form expressions for Kendall's $\tau$ and Spearman's $\rho$ are available. They need to be evaluated numerically.

4. For the BK-FGM (Type III) copula

- $\quad$ Kendall's $\tau$ will be

$$
\tau_{s}(X, Y)=4\left(1-\frac{\Gamma(1+1 / a) \Gamma(1+b)}{\Gamma(1+1 / a+b)}-\left(1-\frac{\Gamma(1+1 / a) \Gamma(1+b)}{\Gamma(1+1 / a+b)}\right)^{2}\right)-1
$$

(by straightforward integration).

- Spearman's correlation coefficient will be

$$
\rho_{s}(X, Y)=12\left(1-\frac{\Gamma(1+1 / a) \Gamma(1+b)}{\Gamma(1+1 / a+b)}-\left(1-\frac{\Gamma(1+1 / a) \Gamma(1+b)}{\Gamma(1+1 / a+b)}\right)^{2}\right)-3
$$

\section{Dependence Properties}

In this section, we focus on the following properties.

\section{Tail Dependence Property:}

Let $X$ and $Y$ be two continuous random variables with $X \sim F$, and $Y \sim G$. The upper tail dependence coefficient (parameter) $\lambda_{U}$ is the limit (if it exists) of the conditional probability that $Y$ is greater than $100 \alpha$ th percentile of $G$ given that $X$ is greater than the $100 \alpha$ th percentile of $F$ as $\alpha$ approaches 1:

$$
\lambda_{U}=\lim _{\alpha \uparrow 1} P\left(Y>G^{-1}(\alpha) \mid X>F^{-1}(\alpha)\right) .
$$

If $\lambda_{U}>0$, then $X$ and $Y$ are upper tail dependent and asymptotically independent otherwise. Similarly, the lower tail dependence coefficient is defined as

$$
\lambda_{L}=\lim _{\alpha \downarrow 0} P\left(Y \leq G^{-1}(\alpha) \mid X \leq F^{-1}(\alpha)\right) .
$$

Let $C$ be the copula of $X$ and $Y$. Then, equivalently, we can write $\lambda_{L}=\lim _{u \downarrow 0} \frac{C(u, u)}{u}$ and $\lambda_{U}=\lim _{u \downarrow 0} \frac{\tilde{C}(u, u)}{u}$, where $\tilde{C}(u, u)$ is the corresponding joint survival copula given by

$$
\tilde{C}(u, u)=1-2 u+C(u, u) .
$$

Next, we consider the following.

- In our case (for the bivariate KW-FGM (type I) copula model),

$$
\begin{aligned}
\lambda_{L} & =\lim _{u \downarrow 0} \frac{C(u, u)}{u} \\
& =\lim _{u \downarrow 0} u^{2}\left(1+\theta\left(u^{2}\left(1-u^{a_{1}}\right)^{b_{1}}\left(1-u^{a_{2}}\right)^{b_{2}}\right)\right) \\
& =0 .
\end{aligned}
$$

Thus, $X$ and $Y$ are asymptotically independent. The corresponding joint survival copula will be given by

$$
\begin{aligned}
\tilde{C}(u, u) & =1-2 u+C(u, u) \\
& =1-2 u+u^{2}\left(1+\theta\left(u^{2}\left[1-\left((1-u)^{a_{1}}\right)\right]^{b_{1}}\left[1-\left((1-u)^{a_{2}}\right)\right]^{b_{2}}\right)\right) .
\end{aligned}
$$


Again,

$$
\begin{aligned}
\lambda_{U} & =\lim _{u \uparrow 1} \frac{1-2 u+C(u, u)}{1-u} \\
& =\lim _{u \uparrow 1} \frac{2(1-u)}{1-u}-\lim _{u \rightarrow 1} \frac{1-C(u, u)}{1-u} \\
& =2-\lim _{u \uparrow 1} \frac{1-u^{2}\left(1+\theta\left(u^{2}\left[1-\left((1-u)^{a_{1}}\right)\right]^{b_{1}}\left[1-\left((1-u)^{a_{2}}\right)\right]^{b_{2}}\right)\right)}{1-u} \\
& =0 .
\end{aligned}
$$

Thus, $(X, Y)$ are asymptotically dependent.

- For the bivariate KW-FGM (type II) copula model,

$$
\begin{aligned}
\lambda_{L} & =\lim _{u \downarrow 0} \frac{C(u, u)}{u} \\
& =\lim _{u \downarrow 0} u^{2}\left(1+\theta\left(u^{\delta_{1}+\delta_{2}}(1-u)^{2-\left(\delta_{1}+\delta_{2}\right)}\right)\right) \\
& =0,
\end{aligned}
$$

provided $2>\delta_{1}+\delta_{2}$. Hence, it is asymptotically independent provided $2>\delta_{1}+\delta_{2}$.

Again,

$$
\begin{aligned}
\lambda_{U} & =\lim _{u \uparrow 1} \frac{1-2 u+C(u, u)}{1-u} \\
& =\lim _{u \uparrow 1} \frac{2(1-u)}{1-u}-\lim _{u \rightarrow 1} \frac{1-C(u, u)}{1-u} \\
& =2-\lim _{u \uparrow 1} \frac{1-u^{2}\left(1+\theta\left(u^{\delta_{1}+\delta_{2}}(1-u)^{2-\left(\delta_{1}+\delta_{2}\right)}\right)\right)}{1-u} \\
& =0,
\end{aligned}
$$

provided $\delta_{1}+\delta_{2}<2$, this again implying that $(X, Y)$ are asymptotically dependent.

Similarly, one can establish these properties for the bivariate KW-FGM (type III) and (type IV) copula models.

\section{Positive Quadrant Dependent (PQD) and Left-Tail Decreasing (LTD) Property:}

According to Amblard and Girard (2002), (Theorem 3), for $\theta>0$ and $(X, Y)$ a random pair with copula $C(u, v)$ as defined in equation (2), we have the following result:

- $\quad X$ and $Y$ are PQD if and only if either $\forall u \in(0,1)$ and $\forall u \in(0,1), \Phi(u)[\Psi(v)] \geq 0$ or $\Phi(u)[\Psi(v)] \leq 0$,

- $\quad X$ and $Y$ are LTD if and only if $\frac{\Phi(u)}{u}$ and $\frac{\Psi(v)}{v}$ is monotone. Next, consider the following:

Proposition 2. The BK-FGM (Type I, Type II and Type III) copulas are PQD.

Proof. For the modified BK-FGM (Type I) copula, we have $\Phi(u)=u^{a_{1}}\left(1-u^{a_{1}}\right)^{b_{1}}$ and $\Psi(v)=v^{a_{2}}\left(1-v^{a_{2}}\right)^{b_{2}}$. Note that, for any real $\left(a_{1}, a_{2}, b_{1}, b_{2}\right)>0, \Phi(u) \geq 0$, for all $u \in(0,1)$ as well as $\Psi(v) \geq 0$, for all $v \in(0,1)$. Hence, $(X, Y)$ are PQD.

Similarly, one can easily check the PQD property for the other two copula models. 
Proposition 3. The BK-FGM (Type I and Type III) copula exhibits LTD properties, while, for the BK-FGM (Type II), it is indeterministic.

Proof. For the modified BK-FGM (Type I) copula, consider the ratio $\frac{\Phi(u)}{u}=u^{a_{1}}\left(1-u^{a_{1}}\right)^{b_{1}}$. It is monotonically decreasing provided, $a_{1}>1$ and for any $b_{1}>0$, and it is also true for any $u \in(0,1)$. Similar results hold for the other ratio $\frac{\Psi(v)}{v}$, for any $v \in(0,1)$. Hence, it is LTD for only $a_{1}>1$ and for any $b_{1}>0$, but not for any other possible choices of the constants $a_{1}$ and $b_{1}$.

Again, for the modified BK-FGM (Type III)copula, the ratio $\frac{\Phi(u)}{u}=\log (1+(1-u))$. It is monotonically decreasing for any $u \in(0,1)$. Similar results will hold for the other ratio $\frac{\Psi(v)}{v}$, for any $v \in(0,1)$. Hence, it is LTD.

However, for the modified BK-FGM (Type II) copula, these ratios are not uniformly increasing and/or decreasing. This is why it is indeterministic in this sense.

\section{Simulation from a Bivariate Copula}

There are several different methods (for example, acceptance-rejection sampling for bivariate cases, via transformation to a known bivariate distribution, etc.) that are available to simulate/generate bivariate random samples from a bivariate copula. We can, in principle, use the following result Joe (1997), to simulate random samples from our modified BK-FGM type copula as follows. Let us define the conditional copula distribution function (say, of $V$ given $U=u), C_{2 \mid 1}(v \mid u)=\frac{\partial C(u, v)}{\partial u}$. Next, if $U$ and $W$ are independent $U(0,1)$ random variables, then $(U, V)=\left(U, C_{2 \mid 1}^{-1}(W \mid U)\right)$ will have the distribution $C(u, v)$. This method, sometimes known as conditional distribution approach or iterative conditioning, is appealing because it involves only univariate simulation. In our case, we do have closed form expressions of $C_{2 \mid 1}(v \mid u)$ for both types of modified BK-FGM bivariate copula available. For example, for the modified FGM BK (type I) copula, one can write (from Equation (10))

$$
\begin{aligned}
C_{2 \mid 1}(v \mid u) & =\frac{\partial C(u, v)}{\partial u} \\
& =2 \theta u v^{2}\left(\left(1-u^{a_{1}}\right)^{b_{1}}\right)\left(v\left(1-v^{a_{2}}\right)^{b_{2}}\right)+v\left\{1-\theta b_{1} u^{a_{1}}\left(\left(1-u^{a_{1}}\right)^{b_{1}}\right)\left(v\left(1-v^{a_{2}}\right)^{b_{2}}\right)\right\} .
\end{aligned}
$$

Consequently, we can easily apply this method. Needless to say, there are other distinct sampling procedures that are also available (for example, importance sampling, adaptive acceptance-rejection sampling, etc.), which is suitable for other classes of copulas.

\section{Applications}

\subsection{Application in Risk Management}

In practice, several risk managers employ VaR (Value at Risk) as a tool of risk measurement. Briefly speaking, $\mathrm{VaR}$ is the maximal potential loss of a position or a portfolio on some investment horizon at a given confidence level. Because of the enormous literature, we only provide its definition. Let $\left\{P_{t}\right\}_{t=1}^{n}$ be the market values of an asset or a portfolio of assets over $n$ periods, and $X_{t}=-\log \left(\frac{P_{t}}{P_{t-1}}\right)$ be the negative log return (loss) over the $t$-th period. Next, given a positive value $\alpha$ close to 0 , the VaR of $X$ at confidence level $(1-\alpha)$ is given by

$$
V a R=\inf \{x \in \mathbb{R} \mid P(X \leq x) \geq 1-\alpha\} .
$$

For a detailed study on the computation of VaR used in the pure copula method, an interested reader is suggested to see Ouyang et al. (2009). Here, we will propose one idea based on bivariate KW-FGM copula (Type II). We list the steps as follows:

1. Simulate $U, V$ and $W$ independently from standard uniform distribution,

2. If $U \leq \lambda_{s}$, for the given bivariate KW-FGM (Type II) copula (say, $C_{\rho_{s}, 1}$ ), take $(X, Y)^{T}=\left(F_{1}^{-1}(V), F_{2}^{-1}\left(C_{\rho_{s}, 1, U}^{-1}(W)\right)\right)^{T}$. 
3. If $U>\lambda_{s}$, for the given bivariate KW-FGM (Type II) copula (say, $C_{\rho_{s}, 2}$ ), take $(X, Y)^{T}=\left(F_{1}^{-1}(V), F_{2}^{-1}\left(C_{\rho_{s}, 2, U}^{-1}(W)\right)\right)^{T}$.

Then, the random vector $(X, Y)$ has the joint distribution

$$
\tilde{F}(x, y)=\lambda_{s} C_{\rho_{s}, 1}\left(F_{1}(x), F_{2}(y)\right)+\left(1-\lambda_{s}\right) C_{\rho_{s}, 2}\left(F_{1}(x), F_{2}(y)\right),
$$

where $\lambda_{s}=\frac{\rho_{s, 2}-\rho_{s}}{\rho_{s, 2}-\rho_{s, 1}}$, and its marginal distributions are $F_{1}$ and $F_{2}$, and linear correlation is $\rho_{s}$. After this, we consider the following formula $R=-\log \left(\lambda_{1} \exp \left(X_{1}\right)+\lambda_{2} \exp \left(X_{2}\right)\right)$ to generate the random number of the negative log returns of portfolios. Here, $\lambda_{1}$ and $\lambda_{2}$ are the weights and must satisfy $\lambda_{1}+\lambda_{2}=1$. Then, $V a R_{\alpha}$ will be computed by calculating the $(1-\alpha)$-th quantile of $R$.

For illustrative purposes, we consider the portfolio composed of Nasdaq and S\&P 500 stock indices. The database contains 2972 daily closing prices from 2 January 1992 to 1 October 2003 . We denote the log-returns of Nasdaq as variable 1 (X, say) and the log-returns of S\&P 500 as variable $2(Y)$. For details on this data set, see Palaro and Hotta (2006).

From Table 1, it is evident that the annualized means of both series are positive. Both return series distributions are nearly symmetric and have large kurtosis, with the Nasdaq presenting the larger one. We do not present the autocorrelation functions of the series, but, for the Nasdaq returns, only the autocorrelations of lag 12 and 13 are significant at the $5 \%$ level (t statistic equals to 3.68 and 4.48 , respectively). There is no significant correlation for the S\&P 500 returns at the 5\% level. In order to specify the bivariate model for these two returns, and to estimate the associated Var under several bivariate copula models, we will consider some specific Autoregressive integrated moving average-Generalized Autoregressive Conditional Heteroskedastic (or in short, ARMA-GARCH) models, the reason being that return series are usually successfully modeled by ARMA-GARCH models by many authors. As suggested in Palaro and Hotta (2006), we will mainly consider three different ARMA-GARCH models: GARCH-N, GARCH-t, and GARCH-E. In terms of modeling the dependence between the two series, we consider three copula functions that are quite popular among other authors: FGM, Gumbel-Hougaard, Bivariate Gaussian copula along with our bivariate KW-FGM type copulas. In order to asses the accuracy of the VaR estimates at 95\%, and 99\% confidence level, we followed the procedure as discussed in Palaro and Hotta (2006). In the table below, we present the proportion of observations (in brackets), for $t=751$ to 2971, where the portfolio loss exceeded the estimated VaR for $\alpha=0.05$.

Table 1. Descriptive statistics of daily log-returns of Nasdaq and S\&P 500 stock indices.

\begin{tabular}{ccc}
\hline Statistics & Nasdaq & S\&P 500 \\
\hline Mean & 0.00038 & 0.00030 \\
Mean (annualized) & $10.141 \%$ & $7.857 \%$ \\
Standard Deviation & 0.01694 & 0.01076 \\
Minimum & -0.10168 & -0.07113 \\
Median & 0.00122 & 0.00028 \\
Maximum & 0.13255 & 0.05574 \\
Excess of Kurtosis & 4.91481 & 3.78088 \\
Asymmetry & 0.01490 & -0.10267 \\
\hline
\end{tabular}

From Table 2, it appears that the Bivariate KW-FGM (Type III) copula model provided a better result in estimating VaR. This is quite expected, since, for the data, the estimated coefficients $a_{1}$ and $a_{2}$ for the Bivariate KW-FGM (Type III) copula appear to be very close to 1 , which then behaves more like a symmetric copula. In addition, for this data, both of the return series are nearly symmetric. 
Table 2. Proportion of observations (number of observations in brackets), for $t=751$ to 2971 , where the portfolio loss exceeded the estimated Value at Risk for $\alpha=0.05$.

\begin{tabular}{cccc}
\hline Copula & GARCH-N & GARCH-t & GARCH-E \\
\hline Nelsen-Ten & $0.0675(167)$ & $0.0698(122)$ & $0.0322(63)$ \\
Gumbel-Hougaard & $0.0666(128)$ & $0.0207(46)$ & $0.0312(69)$ \\
Bivariate Gaussian & $0.0693(117)$ & $0.0359(92)$ & $0.0281(78)$ \\
Bivariate KW-FGM (Type I) copula & $0.0828(82)$ & $0.0244(42)$ & $0.0206(46)$ \\
Bivariate KW-FGM (Type II) copula & $0.2141(77)$ & $0.0286(48)$ & $0.0153(52)$ \\
Bivariate KW-FGM (Type III) copula & $0.0287(37)$ & $0.0126(30)$ & $0.0103(28)$ \\
Bivariate KW-FGM (Type IV) copula & $0.1354(54)$ & $0.0329(47)$ & $0.0189(39)$ \\
\hline
\end{tabular}

\subsection{An Application to Insurance Data}

Here, we consider one application for the four proposed bivariate KW copula models to a heavily used data set, originally considered by Genest et al. (2009), as well as in Ghosh and Ray (2016). This data set contains two variables:

- $X_{1}$ : an indemnity payment,

- $\quad X_{1}$ : an allocated loss adjustment expense (comprising lawyers' fees and claim investigation process).

This data set is comprised of 1500 general liability claims. Several other authors, among others, have used (for e.g., Chen and Fan (2005)) this data set to demonstrate copula-model selection and fitting in an insurance context. We conjecture that this data might well be explained by one or more bivariate Kumaraswamy copula models derived in this paper. For the sake of simplicity, we apply all four bivariate Kumaraswamy copula models to 1466 uncensored claims. As suggested by Genest et al. (2009), based on a comparative study on the numerical estimates of the dependence parameter $(\theta)$, this imposed restriction has a very little or no effect on it. For the uncensored sample, the observed value of Kendall's tau is 0.4328 . In the table below, we provide results of the goodness-of-fit tests based on the statistics $S_{n}, T_{n}$, and $S_{\xi}$, with $\xi=0$. For a detailed description on each of these goodness-of-fit statistics, see Genest et al. (2009).

Here, the dependence parameter $\theta$ is estimated in each case through inversion of Kendall's $\tau$. The critical values and $p$-values reported in Table 1 are based on $N=30,000$ repetitions of the parametric bootstrap procedure discussed in Genest et al. (2009). From Table 3, it appears that bivariate Kumaraswamy (Type III and Type IV) copula provide a better fit as compared to other BK copula models.

Table 3. Goodness of fit statistics for the insurance data.

\begin{tabular}{ccccccc}
\hline Bivariate Copula & $\boldsymbol{\theta}$ & $\boldsymbol{S}_{\boldsymbol{n}}$ & $\boldsymbol{T}_{\boldsymbol{n}}$ & $\boldsymbol{S}_{\mathbf{0} \boldsymbol{n}}$ & $\boldsymbol{p}$-Value (in \%) & Critical Value $\left(\boldsymbol{c}_{\mathbf{2} \boldsymbol{n}}\right)$ \\
\hline Bivariate KW-FGM (Type I) copula & 0.623 & 3.0755 & 2.643 & 1.036 & 45.3 & 0.422 \\
Bivariate KW-FGM (Type II) copula & 1.233 & 2.189 & 3.547 & 0.427 & 0.18 & 0.163 \\
Bivariate KW-FGM (Type III) copula & 1.026 & 0.147 & 0.564 & 0.117 & 78.3 & 0.795 \\
Bivariate KW-FGM (Type IV) copula & 0.342 & 0.422 & 0.642 & 0.137 & 88.2 & 0.831 \\
FGM copula & 0.589 & 1.567 & 2.034 & 0.493 & 37.2 & 0.327 \\
Nelsen-Ten & 1.253 & 2.384 & 4.031 & 0.622 & 43.4 & 0.285 \\
Gumbel-Hougaard & 0.783 & 1.657 & 2.842 & 0.842 & 18.9 & 0.638 \\
Bivariate Gaussian & 0.732 & 1.268 & 2.416 & 0.715 & 44.8 & 0.483 \\
\hline
\end{tabular}

\section{Conclusions}

In this paper, we consider a modified version of the FGM family of copulas and study some important structural properties including the dependence structure. With this modified version, we consider the construction of bivariate KW distributions and discuss some of their structural properties. It is evident from Equation (2), that, depending on suitable choices of $\Phi()$ and $\Psi($ ) functions, satisfying associated boundary conditions as mentioned earlier, one can generate a plethora of such copula models and subsequently develop a wide spectrum of bivariate KW distributions. Our future work would focus on the following:

- Extension to the multivariate case and study several associated properties. It is noteworthy to mention that, albeit complex nature of these type of models (involving several parameters), we expect that multivariate KW 
distribution construction via such type of copula models will be much more interesting and computationally will be more easy to handle.

- For modeling large losses, asymmetric copulas are more useful as compared to symmetric copulas. Thus, we will consider a family of asymmetric copulas as introduced in Nelsen (2006), Chapter 4, which has the following form:

$$
C(u, v)=u v+\theta a(u) b(v), \quad \theta \in[-1,1] .
$$

Here, $a$ and $b$ are functions defined on the interval $(0,1)$. The associated several types of dependence measures will also be considered. In addition, based on this, bivariate and subsequently multivariate KW distributions construction will be considered and then a comparison study will be made with those bivariate and multivariate KW models constructed under a symmetric class of copulas.

- Since a convex combination of any two (or more) valid copulas is also a copula, we would be interested in studying the role of such a mixture of copula in developing bivariate, and sub- sequently multivariate, Kumaraswamy type distributions. For example, one may start with the following:

$$
C^{\text {mixture }}(u, v)=\theta_{1} C^{\text {symmetric }}(u, v)+\left(1-\theta_{1}\right) C^{\text {asymmetric }}(u, v)
$$

for $\theta_{1} \in(0,1]$.

- A natural multivariate extension of the above asymmetric copula would be

$$
C\left(u_{1}, u_{2}, \cdots, u_{p}\right)=\prod_{i=1}^{p} u_{1}+\theta \prod_{i=1}^{p} a_{i}\left(u_{i}\right),
$$

with $\left(u_{1}, u_{2}, \cdots, u_{p}\right) \in[0,1]^{p}, \quad \theta \in[-1,1]$. A natural question would be what judicious choices of the functions $a_{i}()$, for $i=1,2, \ldots, p$ would result in a tractable model. Associated model inference will be a challenging task due to the involvement of so many parameters. We plan to report all of these findings in a separate article somewhere else.

Acknowledgments: The author would like to thank two anonymous referees for their insightful comments and suggestions, which have greatly helped to improve on an earlier version of this manuscript.

Conflicts of Interest: The author declares no conflict of interest.

\section{References}

Amblard, Cécile, and Stéphane Girard. 2002. Symmetry and dependence properties within a semiparametric family of bivariate copulas. Journal of Nonparametric Statistics 14: 715-27.

Arnold, Barry C., and Indranil Ghosh. 2017. Bivariate Kumaraswamy models involving use of Arnold-Ng copulas. Journal of Applied Statistical Science 22: 227-41.

Bairamov, Ismihan G., and Samuel Kotz. 2000. On a New Family of Positive Quadrant Dependent Bivariate Distribution. Technical Report. Washington: The George Washington University.

Bairamov, Ismihan G., Samuel Kotz, and Muhammet Bekci. 2001. New generalized Farlie-Gumbel-Morgenstern distributions and concomitants of order statistics. Journal of Applied Statistics 28: 521-36.

Balakrishnan, Narayanaswamy, and Chin-Diew Lai. 2009. Continuous Bivariate Distributions, 2 nd ed. New York: Springer.

Barreto-Souza, Wagner, and Artur J. Lemonte. 2013. Bivariate Kumaraswamy distribution: Properties and a new method to generate bivariate classes. Statistics 47: 1-22.

Chen, Xiaohong, and Yanqin Fan. 2005. Pseudo-likelihood ratio tests for model selection in semiparametric multivariate copula models. The Canadian Journal of Statistics 33: 389-414.

Farlie, Dennis J. G. 1960. The performance of some correlation coefficients for a general bivariate distribution. Biometrika 47: 307-23.

Ghosh, Indranil, and Samik Ray. 2016. Some alternative bivariate Kumaraswamy type distributions via copula with application in risk management. Journal of Statistical Theory and Practice 10: 693-706.

Genest, Christian, Michael Gendron, and Michael Bourdeau-Brien. 2009. The Advent of Copulas in Finance. The European Journal of Finance 15: 609-18.

Gumbel, Emil J. 1960. Bivariate exponential distributions. Journal of American Statistical Association 55: 698-707. 
Huang, Jian Shan, and Samuel Kotz. 1999. Modifications of the Farlie-Gumbel-Morgenstern distributions: A tough hill to climb. Metrika 49: 307-23.

Joe, Harry. 1997. Multivariate Models and Multivariate Dependence Concepts. New York: Chapman \& Hall/ CRC Monographs on Statistics \& Applied Probability.

Jones, Chris. 2009. Kumaraswamy's distribution: A beta-type distribution with some tractability advantages. Statistical Methodology 6: 70-81.

Kumaraswamy, Poondi. 1980. Generalized probability density-function for double-bounded random-processes. Journal of Hydrology 462: 79-88.

Lai, Chin-Diew, and Min Xie. 2000. Stochastic Ageing and Dependence for Reliability. New York: Springer.

Morgenstern, David. 1956. Einfache Beispiele zweidimensionaler Verteilungen. Mitteinlings fu Mathematische Statistik 8: 234-35.

Nelsen, Roger. 2006. An Introduction to Copulas. New York: Springer.

Ouyang, Zi-Sheng, Hui Liao, and Xiang-qun Yang. 2009. Modeling dependence based on mixture copulas and its application in risk management. Applied Mathematics-A Journal of Chinese Universities 24: 393-401.

Palaro, Helder P., and Luiz Koodi Hotta. 2006. Using Conditional Copula to Estimate Value at Risk. Journal of Data Science 4: 93-115.

Rodriguez-Lallena, Jose Antonio, and Manuel Ubeda-Flores. 2004. A new class of bivariate copulas. Statistics and Probability Letters 66: 315-25.

Sklar, Abe. 1959. Fonctions de Repartition 'a n Dimensions et Leurs Marges. Publications de I'Institut de Statistique de I'Universite de Paris 8: 229-31.

(C) 2017 by the author. Licensee MDPI, Basel, Switzerland. This article is an open access article distributed under the terms and conditions of the Creative Commons Attribution (CC BY) license (http:/ / creativecommons.org/licenses/by/4.0/). 